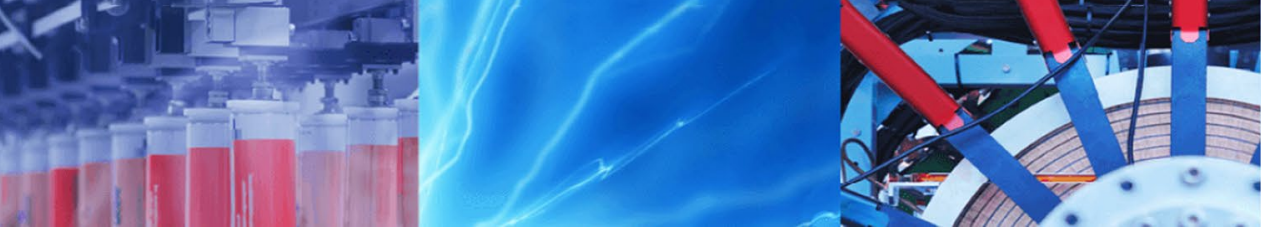

Research Article

\title{
Accurate identification of pavement materials that are susceptible to moisture damage with the use of advanced conditioning and test methods and the use of machine learning techniques
}

\author{
Ram Kumar Veeraragavan ${ }^{1} \cdot$ Nivedya M. K. ${ }^{1}$ Rajib B. Mallick ${ }^{1}$
}

(c) Springer Nature Switzerland AG 2018

\begin{abstract}
In order to prevent premature failure in hot mix asphalt (HMA) pavements due to moisture ingress, it is necessary to identify mixes that are susceptible to moisture damage during the mix design stage. The objective of this paper is to present a method that utilizes a conditioning process along with suitable destructive and nondestructive tests and machine learning techniques to accurately assess the moisture damage potential of HMA mixtures. The scope of work included conditioning with the Moisture Induced Stress Tester (MiST) and testing of a set of HMA mixes with known field performance, and analysis of the data using machine learning techniques. Input variables obtained from a destructive test, the indirect tensile strength test and a non-destructive test, the ultrasonic pulse velocity test, were used to identify the good and poor performing mixes. Various machine learning models were used and it was found that the Support Vector Machine and the Naïve Bayes methods were able to classify the mixes with high accuracy. Use of indirect tensile strength, ultrasonic pulse velocity test and classification based machine learning technique are recommended for the development of accurate models and research in pavement engineering.
\end{abstract}

Keywords Moisture damage $\cdot$ Non-destrcutive tests $\cdot$ MiST $\cdot$ Machine learning

\section{Introduction}

Moisture in any form combined with traffic and environmental conditions can cause significant loss in asphalt pavement strength and durability. Moisture related damage in pavements has been observed since the late 1920s [1]. Extensive research conducted by Hicks [2] showed that about half of the states in United States had experienced moisture-related distresses. Moisture induced damage has been regarded as one of the leading causes of premature failures in asphalt pavements and a major concern to the paving industry.

Over the years, extensive research has been conducted to develop a reliable and practical laboratory test procedure that can simulate field moisture damage condition and which can give predictions that correlate well with field performance. From the literature, it can be concluded that no single laboratory test method can be used to accurately predict the performance of a given hot mix asphalt (HMA) mixture due to various potential mechanisms of moisture damage. Research conducted by the Maine Department of Transportation (MDOT) and other agencies have found the moisture conditioning of HMA samples with the Moisture Induced Stress Tester (MiST) and the use of Indirect Tensile Strength test (ITS) to be effective in screening moisture susceptible HMA mixtures [3]. However, the results of tests were not found to be sufficiently accurate to justify the use of the MiST plus ITS procedure on a regular basis.

This paper presents a study that utilized the MiST and the ITS along with a suitable non-destructive test, the Ultrasonic Pulse Velocity (UPV) test and machine learning

\footnotetext{
$\triangle$ Nivedya M. K., nivedyamk@gmail.com; Ram Kumar Veeraragavan, rveeraragavan@wpi.edu; Rajib B. Mallick, rajib@wpi.edu| ${ }^{1}$ Department of Civil and Environmental Engineering, Worcester Polytechnic Institute, Worcester, MA 01609, USA.
} 
techniques to accurately assess the moisture damage potential of HMA mixtures.

\section{Literature review}

Moisture in liquid or vapor can infiltrate an asphalt pavement through various sources like rain water from the surface through cracks, or by capillary action from underground water through interconnecting air-voids or can exist from the mixing process as a result of improper drying of aggregates during mixing process $[4,5]$. Moisture damage in asphalt pavement has been studied extensively for the past several decades and is defined as the loss in structural strength and durability of an HMA mixture due to the deteriorating effects of moisture [6]. It is a complex phenomenon involving many factors resulting in loss of stiffness and structural strength of the pavement [7]. The moisture by itself does not cause much damage to an asphalt pavement but it is the moisture along with traffic and environmental conditions that accelerates the pavement deterioration process $[8,9]$.

Moisture damage mechanism in HMA can be broadly classified into cohesive failure (within binder or mastic) or adhesive failure (between binder/mastic and aggregate) or combination of both in HMA. Some of the major damage mechanism as reported in previous research studies [10-15] include detachment, displacement, spontaneous emulsification, pore pressure-induced damage, hydraulic scour, and effects of environment on aggregate-asphalt system. Some of the theories that have been developed to explain the adhesion of binder to aggregates include surface energy theory [16], chemical bond theory [17] and mechanical interlock theory $[2,18]$. Some of the other significant factors include binder rheology [19], source, their adsorption, polarity, functional groups present and the affinity of asphalt binders to water [20-22], aggregate characteristics $[19,23,24]$ (such as mineralogy, texture, gradation) and mixture properties [5] (such as air voids, film thickness, permeability). Despite understanding the various types of moisture damage mechanism, researchers all around the world are still trying to find a reliable laboratory test and evaluation procedure to screen HMA mixes that are susceptible to moisture damage $[1,25]$.

Over the past several decades many laboratory testing and conditioning process have been developed, but none of them have been widely accepted by state agencies till date. Most of laboratory test methods are performed on loose mixtures or on compacted samples. The earlier loose mixture tests include boiling water test and static immersion test, which were both qualitative tests, and involved subjecting the loose mixtures at elevated and room temperatures for specific time and observing visually for stripping potential. Some of the compacted mix sample test include Indirect Tensile Strength (ITS) and the dynamic modulus, as well as wheel tracking tests (Hamburg). At present, one of the most widely used mix design test procedure to evaluate moisture damage is the AASHTO T283 test [26], which was developed on the basis of the work conducted by Lottman [27]. However, this test method has been reported to have poor correlation with field performance and has been found to be inconsistent with respect to saturation of the samples. In addition, the AASHTO T283 test process does not simulate the pore pressure that is generated under traffic in the presence of moisture. Researchers have also found that the AAHTO T283 test combined with Hamburg test had difficulties in predicting the field performance of pavement that are moderately resistant to moisture (NCHRP 9-34) [28].

Field observation of HMA mixes in areas of pavement with high water saturation and traffic loading have indicated localized and severe stripping of pavement [14, 29, 30]. Traffic loading in the presence of moisture induces pore water pressure, which contributes to premature failures in asphalt pavements [31]. One of the major drawbacks of many laboratory conditioning systems is that they cannot simulate the damage caused by the generation of pore water pressures. To overcome such a problem, the Moisture Induced Stress Tester (MiST) was developed $(2003)[32,33]$ on the basis of work that was originally conducted by Jimenez [34]. MiST is an accelerated moisture conditioning device that simulates the action of repeated traffic loading on a saturated asphalt pavement by applying cyclic pore water pressure on a HMA sample. It has been found in a study that high pore water pressure does exist in the upper layers of HMA in the field [35] and that the MiST is capable of simulating that condition.

In the MiST conditioning process, a sample is placed inside a chamber with a bladder. The chamber is filled with water and then sealed to prevent the presence of any air inside it. The water is then heated to the desired temperature and the bladder is then pressurized and depressurized sequentially over the desired number of cycles to force water through and out of the sample. The sample subjected to this pressurized cycles is then tested, and the results of tests conducted on the conditioned and the unconditioned samples are compared to evaluate the moisture damage potential of the mix.

Traditional statistical models such as linear regression models or significance tests are normally used to relate mix properties to performance or screen poor performing mixes. The main disadvantage of such traditional statistical methods is that they cannot estimate nonlinear and complex relationships accurately. Recent advances in the area of statistics and data science have led to the development of Machine Learning (ML) techniques [36]. ML techniques 
can be used to predict HMA mix properties more accurately than traditional statistical approaches [37]. There is a need for research on accurate prediction of mix performance using $\mathrm{ML}$ techniques.

Machine learning techniques can be broadly classified into two categories on the basis of technique that are used to train the model: supervised and unsupervised [36]. Supervised learning is the process of training the machine learning model with inputs/features and corresponding targets. In unsupervised learning, there is no target data, and the process is used for clustering data on the basis of their features alone. Supervised machine learning can be further divided into classification and regression techniques. In cases where the response variable (target variable to be predicted) is discrete/categorical, classification techniques are normally used. Regression techniques are used in cases where the response variable is continuous. There are various classification machine learning techniques such as:

- Support vector machines: These models are used for high dimensional and non-linearly separable data. They separate the data by a subspace such as hyperplane capable of separating the observations into two sets.
- Naïve Bayes: These models are used for small data set with a higher number of parameters. The process assumes that the features in a class are not related to each other.

- K-Nearest neighbor:These models are used to establish benchmarks and categorize data based on the classes of their nearest neighbor.

- Decision tree: These models predict the target class by learning some simple decision rules inferred from the features of the entire data.

\section{Materials and methodology}

Twenty-three plant produced loose HMA mixtures of known field performances (by field observation and experience) were utilized in this study. Thirteen loose HMA mixtures, which were provided by one department of transportation (DOT) were compacted with a Superpave gyratory compactor at $5 \pm 1 \%$ voids, and the remaining ten HMA mixtures, which were provided by another DOT were compacted at $7 \pm 1 \%$ voids, each according to their standard construction practice.

Table 1 Mix information

\begin{tabular}{|c|c|c|c|c|c|c|}
\hline $\begin{array}{l}\text { Mix no./per- } \\
\text { formance }\end{array}$ & NMAS (mm) & $\begin{array}{l}\text { Target construc- } \\
\text { tion voids (\%) }\end{array}$ & Binder type & $\begin{array}{l}\text { Percentage } \\
\text { of binder }\end{array}$ & $\begin{array}{l}\text { Percentage } \\
\text { of RAP }\end{array}$ & Additive \\
\hline 1/good & 12.5 & $5 \pm 1$ & PG 64-28 & 5.6 & 20 & No \\
\hline 2/good & 12.5 & $5 \pm 1$ & PG 64-28 & 5.5 & 20 & No \\
\hline 3/poor & 9.5 & $5 \pm 1$ & PG 64-28 & 6.2 & 10 & No \\
\hline 4/good & 9.5 & $5 \pm 1$ & PG 64-28 & 6.4 & 20 & No \\
\hline 5/good & 9.5 & $5 \pm 1$ & PG 64-28 & 6.5 & 20 & Lime \\
\hline 6/good & 12.5 & $5 \pm 1$ & PG 64-28 & 5.3 & 20 & No \\
\hline 7/poor & 9.5 & $5 \pm 1$ & PG 64-28 & 6.5 & 0 & No \\
\hline 8/poor & 9.5 & $7 \pm 1$ & PG 58-28 & 6 & 20 & Commercia \\
\hline 9/good & 12.5 & $7 \pm 1$ & PG $70-28^{*}$ & 4.9 & 15 & No \\
\hline 10/good & 12.5 & $5 \pm 1$ & PG 64-28 & 5.6 & 20 & No \\
\hline 11/poor & 9.5 & $7 \pm 1$ & PG 58-28 & 6.0 & 20 & No \\
\hline $12 /$ good & 9.5 & $5 \pm 1$ & PG 64-28 & 6.6 & 20 & No \\
\hline 13/poor & 12.5 & $5 \pm 1$ & PG 64-28 & 5.8 & 0 & Lime \\
\hline 14/good & 12.5 & $5 \pm 1$ & PG 64-28 & 5.8 & 20 & No \\
\hline 15/good & 12.5 & $5 \pm 1$ & PG 64-28 & 6.0 & 10 & No \\
\hline 16/good & 12.5 & $5 \pm 1$ & PG 64-28 & 5.7 & 20 & No \\
\hline 17/good & 12.5 & $5 \pm 1$ & PG 64-28 & 5.0 & 20 & No \\
\hline 18/poor & 12.5 & $7 \pm 1$ & PG 64-28 & 5.7 & 20 & No \\
\hline 19/poor & 12.5 & $7 \pm 1$ & PG 64-28 & 5.4 & 10 & No \\
\hline 20/good & 12.5 & $7 \pm 1$ & PG 64-28 & 4.9 & 20 & No \\
\hline $21 /$ poor & 12.5 & $7 \pm 1$ & PG 64-28 & 4.6 & 20 & No \\
\hline $22 /$ poor & 12.5 & $7 \pm 1$ & PG 64-22 & 5.0 & 0 & No \\
\hline 23/good & 12.5 & $7 \pm 1$ & PG 64-28 & 5.4 & 10 & No \\
\hline
\end{tabular}

*Polymer modified 
Table 1 presents the different properties of each mix. A minimum of three samples were used for each test, for each mix.

The compacted samples were then subjected to moisture conditioning using the MiST. MiST conditioning was conducted according to the ASTM D7870 standard, which specifies 3500 cycles at $275 \mathrm{kPa}$, and at $60^{\circ} \mathrm{C}$ for PG 64-28 and PG 70-28 mixes, and $50{ }^{\circ} \mathrm{C}$ for PG 58-28 mixes. In addition to this ASTM protocol the samples were subjected to a 20 -hour dwell period (saturation) prior to the application of cyclic pore pressure. The dwell period simulated the soaking period of water immediately after construction before the passage of traffic and allowed the diffusion of water into the asphalt-aggregate interface.

To evaluate the performance of HMA sample before and after MiST conditioning, a non-destructive testing, the Ultrasonic Pulse Velocity test (UPV), and a destructive testing, the Indirect Tensile Strength (ITS) test were used. The UPV testing, which is based on surface wave propagation techniques was conducted according to the ASTM C597-09 standard. The UPV testing works by converting the electrical pulse from a piezoelectric crystal to ultrasonic shock waves ( $p$-waves), which travel from the transducer through the specimen and are then collected by the receiver on the other side of the specimen (direct transmission position). The shock waves received from receiving transducer are converted back to an electrical pulse. The seismic modulus is then calculated from the ultrasonic pulse velocity or the $p$-wave travel time, according to the following equation.

$V_{p}=H / t_{v} ; \quad M_{v}=\rho \times V_{p^{2}}^{2} \quad E_{s}=M_{v} \times \frac{[(1+\mu) \times(1-2 \mu)]}{(1-\mu)}$

$E_{d}=\left(E_{s} / 3.2\right) \times$ temperature correction factor; temperature correction factor $=0.95$ (for a test temperature of $21.1{ }^{\circ} \mathrm{C}$ ); where $V_{p}=$ velocity of wave; $t_{v}=$ time of travel; $\rho=$ density; $\mu=$ Poisson's ratio; $E_{s}=$ seismic modulus, $E_{d}=$ design modulus.

Hence, any change in the material property after MiST testing would be reflected in the ultrasonic travel time. The ITS testing was conducted according to the ASTM D6931 procedure. The results from UPV and ITS testing are shown in Figs. 1 and 2 respectively. In Fig. 1, it can be seen that seismic modulus of moisture conditioned samples were lower than unconditioned samples for all mixes, except for mixes 9 and 15 which are the better performing mixes. This shows that UPV testing is sensitive to the effects of moisture damage. The indirect tensile strength was reduced after moisture conditioning, as can be seen in Fig. 2, irrespective of good or poor performance in the field. A conventional statistical analysis of

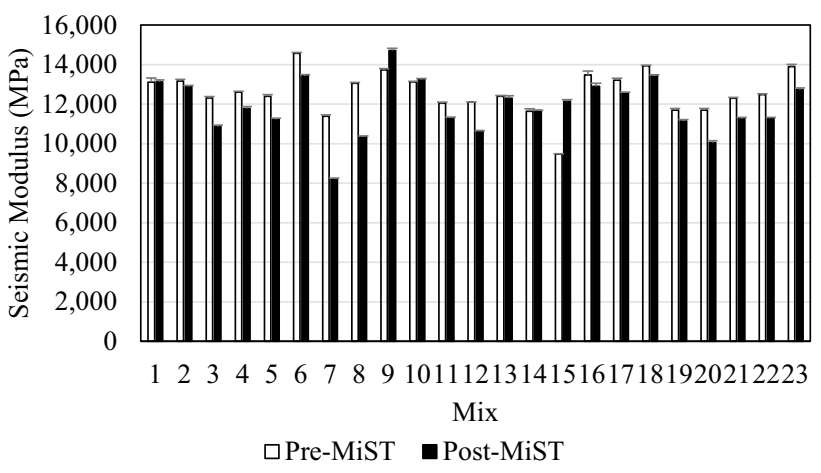

Fig. 1 Seismic modulus values before and after MiST conditioning. Note: The error bars indicate the standard deviation of the data

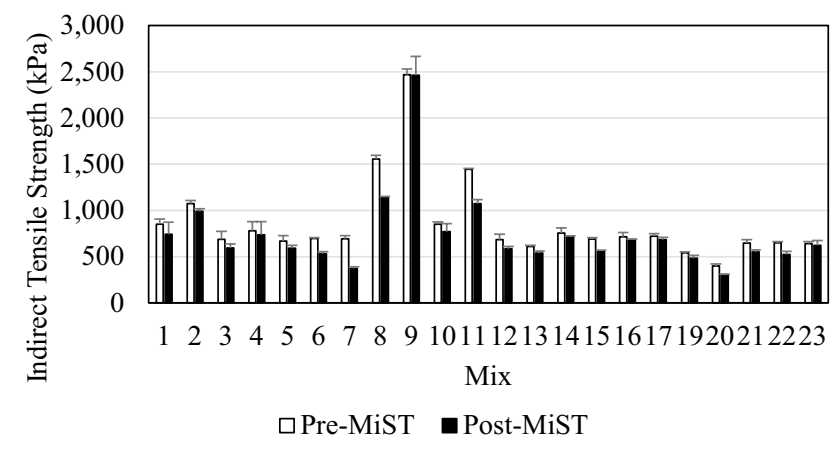

Fig. 2 Indirect Tensile Strength before and after MiST conditioning. Note: The error bars indicate the standard deviation of the data

the data, as shown in Table 2, showed inaccurate identification of good and poor performing mixes-note that some good mixes show significant reduction in modulus/strength. Although some poor mixes showed significant reduction, the overall accuracy of prediction was $82.6 \%$ for $E_{s}$ and $73.9 \%$ for ITS.

Based on the collected laboratory test data, supervised machine learning classification techniques were used to predict the performance (good or poor) of the HMA mixes. Here, post-MiST seismic modulus values and post-MiST ITS values were used as input variables. The K-NN, Naïve Bayes, Support Vector Machines (SVM) and decision tree methods of ML were used in this study. The data consisted of test properties of each sample, and the samples were labelled according to the observed performance (good or poor) of the mix from which the samples were prepared. Therefore, the total dataset consisted of 58 observations. The training and validation data consisted of $65 \%$ and test data consisted of $35 \%$ of the total data. K-fold cross validation was carried out in order to avoid overfitting and the value of $k$ used was five. A confusion matrix is a representation of the error matrix that is generally used to visualize the performance of the machine learning classification models. 
Table 2 Summary of statistical analysis

\begin{tabular}{|c|c|c|c|c|}
\hline Mix & Performance & Additive & $\begin{array}{l}\text { Siesmic modulus difference } \\
\text { significant or not? }\end{array}$ & $\begin{array}{l}\text { ITS difference } \\
\text { significant or } \\
\text { not? }\end{array}$ \\
\hline 1 & Good & No & No & No \\
\hline 2 & Good & No & No & Yes \\
\hline 3 & Poor & No & Yes & No \\
\hline 4 & Good & No & No & No \\
\hline 5 & Good & Lime & Yes & No \\
\hline 6 & Good & No & No & No \\
\hline 7 & Poor & No & Yes & Yes \\
\hline 8 & Poor & Commercial & Yes & Yes \\
\hline 9 & Good & No & No & No \\
\hline 10 & Good & No & No & No \\
\hline 11 & Poor & No & Yes & Yes \\
\hline 12 & Good & No & No & No \\
\hline 13 & Poor & Lime & No & No \\
\hline 14 & Good & No & No & No \\
\hline 15 & Good & No & No & No \\
\hline 16 & Good & No & No & No \\
\hline 17 & Good & No & No & No \\
\hline 18 & Poor & No & Yes & No \\
\hline 19 & Poor & No & Yes & No \\
\hline 20 & Good & No & Yes & Yes \\
\hline 21 & Poor & No & Yes & Yes \\
\hline 22 & Poor & No & No & Yes \\
\hline 23 & Good & No & No & No \\
\hline
\end{tabular}

True positive (TP) identifies the good mixes as good mixes and true negative (TN) identifies the poor mixes as poor mixes and accuracy is calculated as

Accuracy $=\frac{T P+T N}{\text { Total observation }(n)}$

The accuracy for $E_{s}$ is calculated below

$\mathrm{TP}=12 ; \mathrm{TN}=7$

Accuracy $=\frac{12+7}{23}=82.6 \%$

Accuracy for ITS is calculated below

$\mathrm{TP}=12 ; \mathrm{TN}=5$

Accuracy $=\frac{12+5}{23}=73.9 \%$

Confusion matrix was constructed to evaluate the prediction accuracy of the different models. It can be seen in Fig. 3 that Naïve Bayes and SVM based models have the highest accuracy (95\%), much higher than that from the statistical analysis.

\section{Conclusions and recommendations}

The following conclusions and recommendations can be made from the study.

1. MiST conditioning process with the 20-hour dwell period prior to 3500 pressure cycling at $275 \mathrm{kPa}$ was able to simulate field moisture damage conditions in the laboratory.

2. Non-destructive testing using ultrasonic pulse velocity was able to identify moisture susceptible mixes.

3. The test properties such as seismic modulus and indirect tensile strength after moisture conditioning can be used by agencies to predict the moisture damage potential of HMA.

4. Models developed with two machine learning techniques, the Support Vector Machine and Naïve Bayes (NB) method show excellent accuracy for classification of mixes into good and poor performing in terms of potential of moisture damage.

5. The use of the indirect tensile strength test along with the ultrasonic pulse velocity test is recommended. 


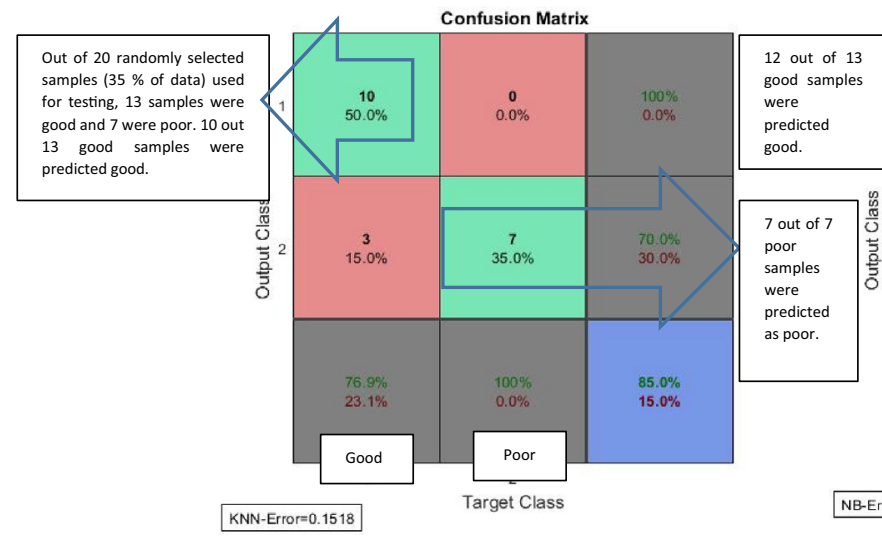

$\mathbf{a}$
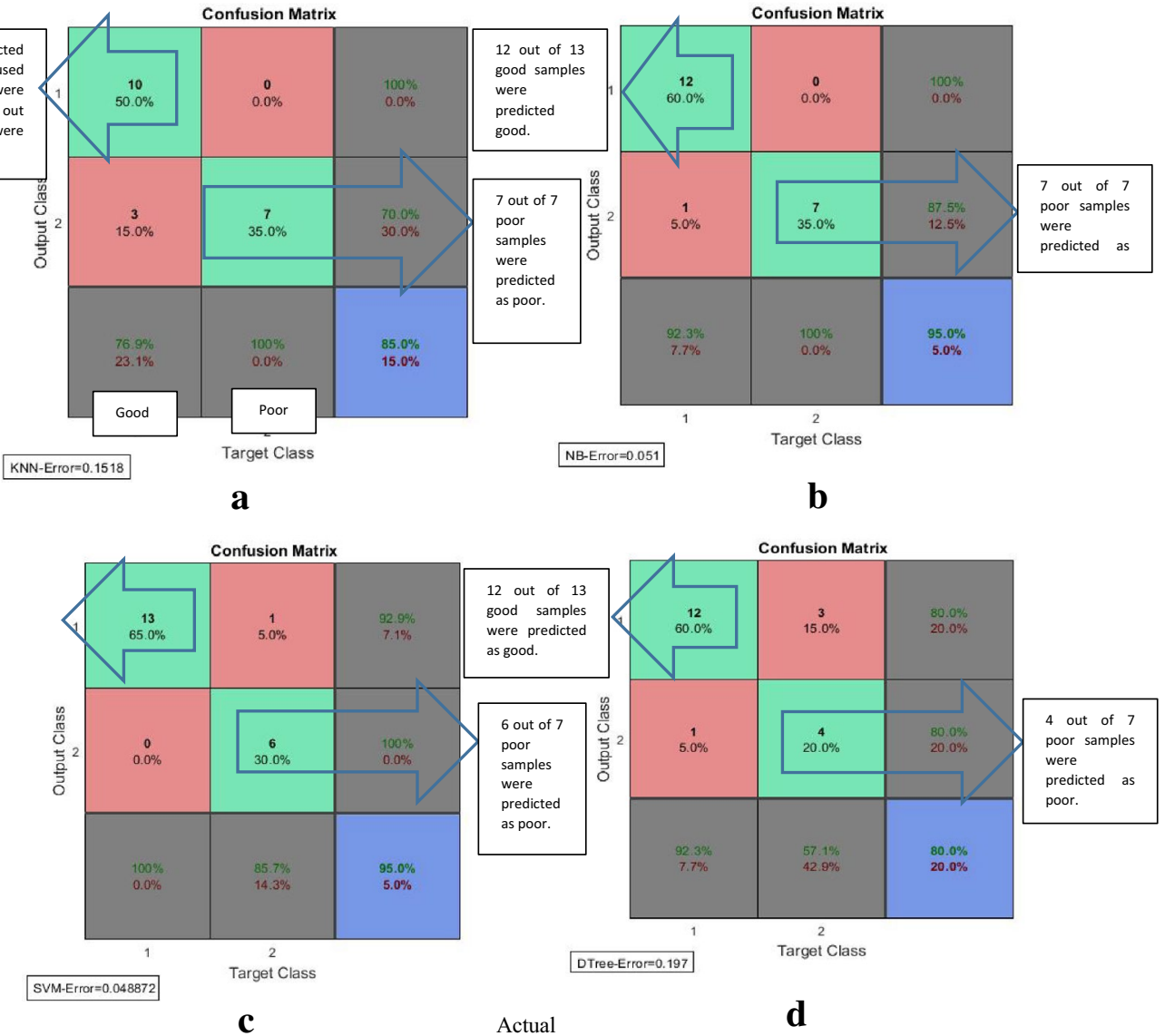

c

Actual

b

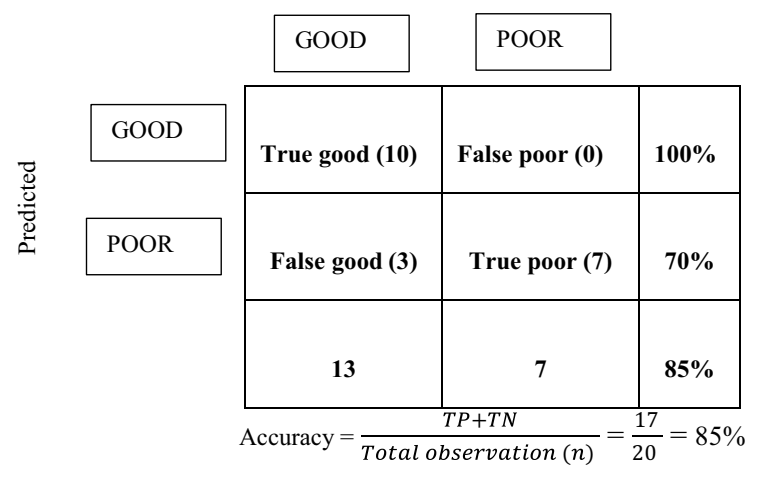

$\mathbf{e}$

Fig. 3 Confusion matrix for different machine learning models with an example calculation of accuracy of K-NN classification model. a Confusion matrix for K-NN classification; Accuracy $=85 \%$. b Confusion matrix for Naïve Bayes classification; Accuracy=95\%. c Con- fusion matrix for SVM classification; Accuracy $=95 \%$. d Confusion matrix for Decision tree classification; Accuracy $=80 \%$. e Example calculation of accuracy of K-NN model
6. Machine learning models should be developed and utilized for accurate classification of HMA mixes according to their moisture damage potential.

7. The use of machine learning methods should be explored for other areas of research in pavement engineering.

\section{SN Applied Sciences}

\section{References}

1. Hamzah MO, Kakar MR, Hainin MR (2015) An overview of moisture damage in asphalt mixtures. Jurnal Teknologi (Sciences \& Engineering) 73(4):125-131 
2. Hicks RG (1991) Moisture damage in asphalt concrete. NCHRP Synthesis of Highway Practice 175, Transportation Research Board, Washington, DC

3. Arepalli UM (2018) A study of moisture induced material loss of hot mix asphalt (HMA). Ph.D. dissertation, Dept. of Civil and Environmental Engineering, Worcester Polytechnic Institute, MA

4. Kringos N, Scarpas A (2008) Physical and mechanical moisture susceptibility of asphaltic mixtures. Int J Solids Struct 45(9):2671-2685

5. Behiry AEAE-M (2013) Laboratory evaluation of resistance to moisture damage in asphalt mixtures. Ain Shams Eng J 4(3):351-363

6. Santucci L (2002) Moisture sensitivity of asphalt pavements. Tech Topics

7. Yi J, Shen S, Wang D, Feng D, Huang Y (2016) Effect of testing conditions on laboratory moisture test for asphalt mixtures. $J$ Test Eval 44(2):856-867

8. Grenfell J, Ahmad N, Liu Y, Apeagyei A, Large D, Airey G (2014) Assessing asphalt mixture moisture susceptibility through intrinsic adhesion, bitumen stripping and mechanical damage. Road Mater Pavement Des 15(1):131-152

9. Zhang J, Apeagyei AK, Airey GD, Grenfell JR (2015) Influence of aggregate mineralogical composition on water resistance of aggregate-bitumen adhesion. Int J Adhes Adhes 62:45-54

10. Kiggundu BM, Roberts FL (1988) Stripping in HMA mixtures: state-of-the-art and critical review of test methods. National Center for Asphalt Technology, Auburn

11. Taylor MA, Khosla NP (1983) Stripping of asphalt pavements: state of the art (discussion, closure)

12. Al-Swailmi S, Terrel R (1994) Water sensitivity of asphalt-aggregate mixtures: test selection, SHRP-A

13. Choubane B, Page G, Musselman J (2000) Effects of water saturation level on resistance of compacted hot-mix asphalt samples to moisture-induced damage. Transp Res Rec J Transp Res Board 1723:97-106

14. Kandhal PS, Rickards I (2001) Premature failure of asphalt overlays from stripping: case histories. Asphalt Paving Technol 70:301-351

15. Little DN, Petersen JC (2005) Unique effects of hydrated lime filler on the performance-related properties of asphalt cements: physical and chemical interactions revisited. J Mater Civ Eng 17(2):207-218

16. Read J, Whiteoak D (2003) The shell bitumen handbook. Thomas Telford

17. Syunyaev R, Balabin R, Akhatov I, Safieva J (2009) Adsorption of petroleum asphaltenes onto reservoir rock sands studied by near-infrared (NIR) spectroscopy. Energy Fuels 23(3):1230-1236

18. Masad E, Castelblanco A, Birgisson B (2005) Effects of air void size distribution, pore pressure, and bond energy on moisture damage. J Test Eval 34(1):1-9

19. Curtis CW, Ensley K, Epps J (1993) Fundamental properties of asphalt-aggregate interactions including adhesion and absorption. National Research Council, Washington, DC

20. Caro S, Masad E, Bhasin A, Little DN (2008) Moisture susceptibility of asphalt mixtures, Part 1: mechanisms. Int J Pavement Eng 9(2):81-98

21. Plancher H, Dorrence S, Petersen J (1977) Identification of chemical types in asphalts strongly adsorbed at the asphalt-aggregate interface and their relative displacement by water. [Moisture damage to roads], Energy Research and Development Administration, Laramie, WY (USA). Laramie Energy Research Center

22. Petersen J, Plancher H, Ensley E, Venable R, Miyake G (1982) Chemistry of asphalt-aggregate interaction: relationship with pavement moisture-damage prediction test. Transp Res Rec (843)

23. Bagampadde U, Isacsson U, Kiggundu B (2005) Influence of aggregate chemical and mineralogical composition on stripping in bituminous mixtures. Int J Pavement Eng 6(4):229-239

24. Airey G, Masad E, Bhasin A, Caro S, Little D (2007) Asphalt mixture moisture damage assessment combined with surface energy characterization. In: Proceedings of a conference on advanced characterization of pavement and soil engineering materials, pp 739-748

25. Solaimanian M, Harvey J, Tahmoressi M, Tandon V (2003) Test methods to predict moisture sensitivity of hot-mix asphalt pavements. In: moisture sensitivity of asphalt pavements-a national seminar California Department of Transportation; Federal Highway Administration; National Asphalt Pavement Association; California Asphalt Pavement Alliance; and Transportation Research Board

26. AASHTO T 283 (2014) Standard method of test for resistance of compacted asphalt mixtures to moisture-induced damage. AASHTO, Washington, DC

27. Lottman RP (1982) Laboratory test methods for predicting moisture-induced damage to asphalt concrete

28. Solaimanian M, Harrigan E (2002) Improved conditioning procedure for predicting the moisture susceptibility of HMA pavements. In: Proceedings of moisture damage symposium, Laramie, Wyoming, USA

29. Kandhal PS, Lubold CW, Roberts FL (1989) Water damage to asphalt overlays: case histories. National Center for Asphalt Technology, Nashville

30. Shakiba M, Darabi MK, Little DN (2017) Effect of pore water pressure on response of asphalt concrete. Transp Res Rec J Transp Res Board 2631:114-122

31. Dawson A (2008) Water in road structures: movement, drainage \& effects. Springer

32. Regimand A, James LH, Muse PD, Landreth K, He T (2012) System and method for conditioning and detection of susceptibility to moisture damage in asphalt mixes. Google Patents

33. Regimand A, James LH, Muse PD, He T (2004) System and method for conditioning and detection of moisture damage in asphalt mixes. Google Patents

34. Jimenez R (1974) Testing for debonding of asphalt from aggregates. Transportation Research Record 515

35. Mallick RB, Gould JS, Bhattacharjee S, Regimand A, James LH, Brown ER (2003) Development of a rational procedure for evaluation of moisture susceptibility of asphalt paving mixes. Transportation Research Board, Washington, DC

36. Bishop CM (2006) Pattern recognition and machine learning. Springer, New York

37. Nivedya MK, Mallick RB (2018) Accurate prediction of laboratory permeability of hot mix asphalt using machine learning techniques, In: Proceedings of Advances in Materials and Pavement Performance Prediction. Doha, Qatar 